\title{
Knockdown of endothelin A receptor expression inhibits osteosarcoma pulmonary metastasis in an orthotopic xenograft mouse model
}

\author{
YONG LI, QIANDE LIAO, KANGHUA LI, DA ZHONG, XIAOJUN WENG and MENG MI \\ Department of Orthopedics, Xiangya Hospital, Central South University, Changsha, Hunan 410008, P.R. China
}

Received November 21, 2011; Accepted February 29, 2012

DOI: $10.3892 / \mathrm{mmr} .2012 .842$

\begin{abstract}
Previous in vitro studies suggest that the endothelin A receptor (ETAR) could be a potential therapeutic target for osteosarcoma (OS) metastasis. In the present study, we assessed for the first time the role of ETAR in OS proliferation and pulmonary metastasis in vivo. MG-63 human OS cells were stably transduced with ETAR shRNA or scramble control shRNA and injected into the tibia of nude mice to generate an orthotopic xenograft OS model. The mice were divided into three groups ( $n=10$ each group): i) the untransduced control group, where the mice were injected with untransduced MG-63 cells; ii) the scramble control group, where the mice were injected with cells stably transduced with the scramble control shRNA; iii) the ETAR-shRNA group, where the mice were injected with cells stably transduced with ETAR shRNA. The ETAR shRNA knocked down more than $75 \%$ of endogenous ETAR expression and significantly inhibited invasion, but not proliferation/viability of MG-63 cells in vitro. In the orthotopic xenograft OS mouse model, no significant difference in the tumor volume was observed over time among the untransduced control, the scramble control and the ETAR-shRNA groups. However, a combination of clinical signs, organ examinations and quantitative clonogenic lung metastasis assays showed that lung metastasis in the ETAR-shRNA group was significantly lower than that in the control groups. Immunohistochemical analyses revealed that the matrix metalloproteinase-2 (MMP-2) expression was significantly reduced in the ETAR-shRNA group compared with the control groups. The results were confirmed with western blot analyses using primary tumor tissues or the stably transduced MG-63 cells. In conclusion, we demonstrated in an orthotopic xenograft OS model that ETAR is critical for OS pulmonary metastasis, but not for tumor growth. This study
\end{abstract}

Correspondence to: Dr Qiande Liao, Department of Orthopedics, Xiangya Hospital, Central South University, 87 Xiangya Road, Changsha, Hunan 410078, P.R. China

E-mail: sunshinexxus@yahoo.com

Key words: endothelin, endothelin A receptor, osteosarcoma, proliferation, pulmonary metastasis, orthotopic xenograft provides the first in vivo evidence suggesting an important role for ETAR in OS pulmonary metastasis.

\section{Introduction}

Osteosarcoma (OS) is the most frequent primary bone malignancy and the eighth most common type of cancer among children, comprising $2.4 \%$ of all malignancies in pediatric patients and approximately $35 \%$ of all bone cancers (1). OS is a devastating disease, characterized by high local aggressiveness and a tendency to metastasize to the lungs and distant bones. In spite of the use of neoadjuvant chemotherapy and improvements in surgical technology that have increased the survival rate to $65-75 \%$, pulmonary metastasis occurs in approximately $40-50 \%$ of OS patients $(2,3)$ and remains a major cause of fatal outcome (4). The cure rate of OS is approximately $65 \%$ for patients with localized diseases. When presenting with metastases at the time of diagnosis, the survival rate is $25 \%$ $(5,6)$. Thus, it is important to identify and confirm potential therapeutic targets involved in OS progression, particularly, pulmonary metastasis.

The endothelin-1 (ET-1)/endothelin A receptor (ETAR) pathway reportedly is involved in a wide range of cancerrelevant processes, such as inhibition of apoptosis, matrix remodeling, bone deposition and metastases (7). ET-1 and ETAR are expressed in OS tissue and cells $(8,9)$. A few in vitro studies have reported that ETAR blockade could inhibit OS cell invasion, suggesting that ETAR could be a potential therapeutic target for OS metastasis $(8,9)$. However, no in vivo evidence has been reported. In the present study, we assessed the role of ETAR in OS proliferation and pulmonary metastasis in vivo, using an orthotopic xenograft OS mouse model.

\section{Materials and methods}

Cell line, reagents and mice. The MG-63 human OS cell line was purchased from the American Tissue Culture Collection (ATCC, Manassas, VA, USA). ETAR shRNA lentiviral particles (sc-39960-V), control shRNA lentiviral particles-A(sc-108080), anti-ETAR (sc-21193) antibody, and anti-matrix metalloproteinase-2 (MMP-2) antibody (sc-53630) (10) were purchased from Santa Cruz Biotechnology, Inc. (Santa Cruz, CA, USA). All secondary antibodies were from Jackson ImmunoResearch 
Laboratories, Inc. (West Grove, PA, USA). Methlythiazol tetrazolium (MTT) cell proliferation and viability assay kit was purchased from R\&D Systems (Minneapolis, MN, USA). Puromycin, collagenase IV, elastase and all chemicals of reagent grade were purchased from Sigma (St. Louis, MO, USA). Fiveweek-old BALB/C female nude mice were purchased from Central South University (Changsha, China) and were housed at the Xiangya Hospital BioResources Centre.

Lentiviral transduction. The ETAR shRNA lentiviral particles contain expression constructs encoding target-specific 19-25 nt (plus hairpin) shRNA designed to specifically knock down ETAR gene expression. The control shRNA lentiviral particles contain a scrambled shRNA sequence that will not lead to the specific degradation of any cellular mRNA, and was used as a negative control for ETAR shRNA lentiviral particles. Lentiviral transduction was performed in MG-63 cells. Pools of stable transductants were generated via selection with puromycin $(5 \mu \mathrm{g} / \mathrm{ml})$ according to the manufacturer's instructions (Santa Cruz Biotechnology, Inc.).

In vitro cell proliferation assay. In vitro cell proliferation and viability were determined with the MTT cell proliferation and viability assay kit as described by the manufacturer $(R \& D$ Systems). Briefly, cells were cultured at $15 \times 10^{3}$ cells/well in 96-well tissue culture plates and incubated at $37^{\circ} \mathrm{C}$ for $24 \mathrm{~h}$. At the end of the culture period, cells were washed with phosphatebuffered saline (PBS), the MTT reagents were added according to the manufacturer's recommendations, and the absorbance was measured at $570 \mathrm{~nm}$ using an ELISA plate reader. The proliferation/viability of cells stably transduced with scramble control shRNA or ETAR-shRNA was shown as fold changes to that of untransduced control cells (designated as 1). Each experiment was repeated for three times in triplicates.

In vitro cell invasion assay. Transwell ${ }^{\circledast}$ cell invasion assay (Corning Life Sciences, Lowell, MA, USA) was performed as previously described (11). Briefly, Transwell cell-culture chambers with an $8-\mu \mathrm{m}$ pore size (BD Biosciences, Bedford, MA, USA) for 24-well plates were coated with $50 \mu 1$ Matrigel (BD Biosciences). MG-63 cells were seeded in the upper chamber at $5 \times 10^{5}$ cells/well in RPMI-1640 serum-free medium. Complete medium $(600 \mathrm{ml})$ was added to the lower chamber. Cells were allowed to migrate for $24 \mathrm{~h}$ followed by fixation and staining with crystal violet. Migrating cells which adhered to the bottom of the filter were counted in 10 random fields/chamber under a microscope. Each experiment was repeated for three times in triplicates.

Establishment of the orthotopic xenograft OS mouse model. MG-63 cells were mixed with 50\% Matrigel to a concentration of $2 \times 10^{6}$ cells $/ \mathrm{ml}$. Mice were anaesthetized by intraperitoneal injection of $100 \mathrm{mg} / \mathrm{kg}$ ketamine and $10 \mathrm{mg} / \mathrm{kg}$ xylazine. A volume of $10 \mu \mathrm{l}$ of MG-63/Matrigel solution was injected into the left tibia of individual mice using a 27 -gauge needle (11). The needle was inserted into the tibial tuberosity and advanced using a drilling motion to avoid fracture of the bone. The mice were divided into three experimental groups $(\mathrm{n}=10$ each group): i) the untransduced control group, where the mice were injected with untransduced MG-63 cells; ii) the scramble control group, where the mice were injected with cells stably transduced with the scramble control shRNA; iii) the ETARshRNA group, where the mice were injected with cells stably transduced with ETAR shRNA. The mice were monitored three times weekly for tumor growth and signs of distress. Tumors were measured in the anteroposterior and lateral planes using digital callipers. Leg volume and tumor volume were calculated using the formula $4 / 3 \pi[1 / 4(\mathrm{AP}+\mathrm{L})]^{2}(12)$. The volume of the contralateral limb was subtracted from the tumor-bearing limb to calculate actual tumor volume. Mice were weighed using digital scales. Tumor growth was evaluated until death or sacrifice when tumor dimensions exceeded $5 \%$ body weight or mice showed dyspnea, abnormal posture, $>20 \%$ body weight loss, difficulty with ambulation, or any other clinical sign of metastatic disease causing significant pain or distress, according to the institutional guidelines. Thirty-five days after MG-63 inoculation, all animals were sacrificed, as the primary tumors had grown to a disabling size. All animal care, breeding, and testing procedures were approved by the Laboratory Animal Users Committee at Xiangya Hospital, Central South University.

Clonogenic lung metastasis assay. Clonogenic lung metastasis assays were performed as previously described (13). Briefly, lungs from each individual animal were minced into 1-mm pieces, and digested with $5 \mathrm{ml}$ enzyme cocktail containing $1 \mathrm{mg} / \mathrm{ml}$ collagenase IV and $6 \mathrm{U} / \mathrm{ml}$ elastase in PBS for $1 \mathrm{~h}$ at $4^{\circ} \mathrm{C}$ with rotation. Cell suspensions were filtered through 70-Am nylon cell strainers and washed two times with Hank's buffered saline, then resuspended in complete medium. The cells were then cultured in $10-\mathrm{cm}$ tissue culture dishes and treated with $5 \mu \mathrm{g} / \mathrm{ml}$ of puromycin to allow only the growth of lentiviral transduced MG-63 cells. When colonies of growing MG-63 cells became visible (8-14 days), the plates were washed with PBS, fixed with methanol, and stained with crystal violet. The colonies were counted independently by two investigators, blinded to the group to which each mouse belonged, and the total colony number/lungs was calculated for each animal.

Immunohistochemistry. Paraffin-embedded tumor tissues were examined for MMP-2 expression. The immunostaining for MMP-2 was performed utilizing the streptavidin-biotin-peroxidase method, according to the manufacturer's instructions (Beijing Golden Bridge Biotechnology, Inc., Beijing, China). In brief, sections ( $4 \mu \mathrm{m})$ of paraffin-embedded specimens were de-paraffinized in xylene, hydrated in a degraded series of ethanol, and heated in $0.01 \mathrm{M}$ citrate buffer for $10 \mathrm{~min}$ in a microwave oven. After cooling for $20 \mathrm{~min}$ and washing in PBS, endogenous peroxidase was blocked with methanol containing $0.3 \%$ hydrogen peroxide for $30 \mathrm{~min}$, followed by incubation with PBS for $30 \mathrm{~min}$. Then the sections were incubated with anti-MMP-2 antibody at a dilution of 1:150, and stained using the avidin-biotin complex method. Coloration was developed by DAB containing $\mathrm{H}_{2} \mathrm{O}_{2}$, and the sections were counter-stained with hematoxylin. The number of positive cells was counted in 10 high-power view fields and a percentage was calculated as follows: MMP-2 positive cells/total tumor cells x100\%.

Western blot analysis. Immunoblotting was performed as described previously with respective antibodies (14). Briefly, 
A

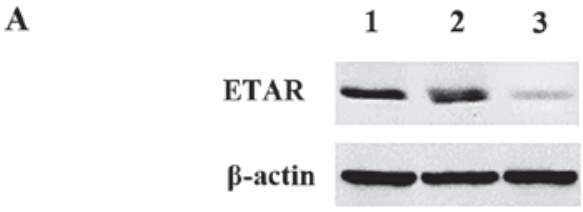

B

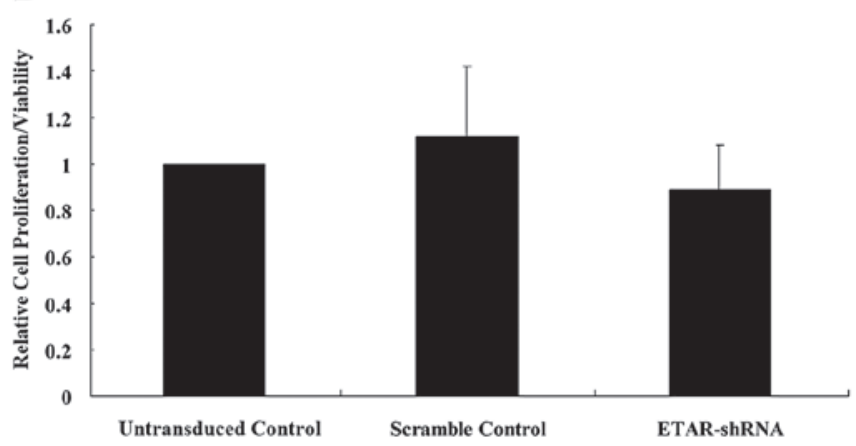

C

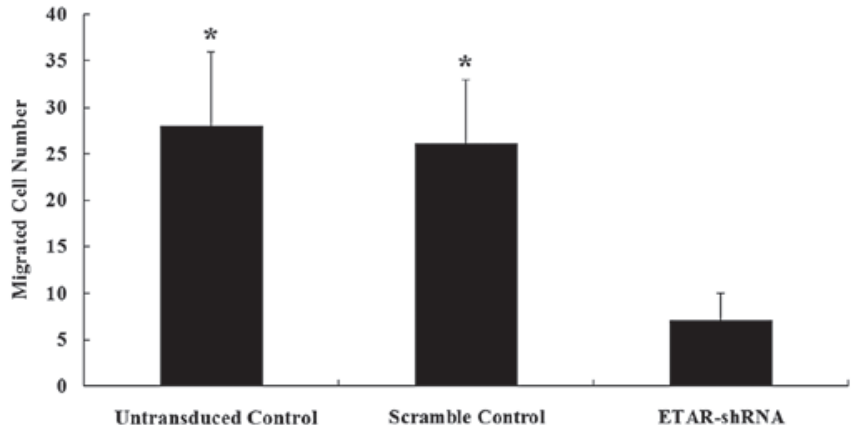

Figure 1. In vitro proliferation/viability and invasion ability in endothelin A receptor (ETAR) knockdown MG-63 osteosarcoma cells. (A) Expression of ETAR in untransduced MG-63 cells (lane 1) and cells stably transduced with scramble control shRNA (lane 2) or ETAR-shRNA (lane 3) was analyzed with western blot analysis. $\beta$-actin blotting was used as a loading control. Representative results are shown in this figure. (B) In vitro cell proliferation/ viability was determined with MTT assays. Proliferation/viability of MG-63 cells stably transduced with scramble control shRNA or ETAR-shRNA was expressed as fold changes to that of untransduced control cells (designated as 1). Results are expressed as mean \pm SD. (C) In vitro cell invasion ability was determined with Transwell cell invasion assays. Migrated cell numbers were counted. Results are expressed as mean $\pm \mathrm{SD}$. ${ }^{*} \mathrm{P}<0.05$ compared with ETAR-shRNA cells.

MG-63 cells or homogenized tumor tissues were lysed in $0.1 \%$ Nonidet P-40 lysis buffer [0.1\% Nonidet P-40, $50 \mathrm{mM}$ Tris- $\mathrm{HCl}$ (pH 7.4), $150 \mathrm{mM} \mathrm{NaCl}$, and $1 \mathrm{mM}$ EDTA]. Equal amounts of proteins for each sample were separated by $10 \%$ SDS-polyacrylamide gels and blotted onto a polyvinylidene difluoride microporous membrane (Millipore, Billerica, MA, USA). Membranes were incubated for $1 \mathrm{~h}$ with a 1/1,000 dilution of anti-ETAR, or anti-MMP-2 antibody, and then washed and revealed using secondary antibodies with horseradish peroxidase conjugate $(1 / 5,000,1 \mathrm{~h})$. Peroxidase was revealed with an GE Healthcare ECL kit. Proteins were quantified before being loaded onto the gel, and equal loading of protein was verified by Ponceau coloration.

Statistical analysis. Statistical analyses were performed with SPSS for Windows 10.0. Data values were expressed as means $\pm \mathrm{SD}$. Comparisons of means among multiple groups were performed with one-way ANOVA followed by post hoc

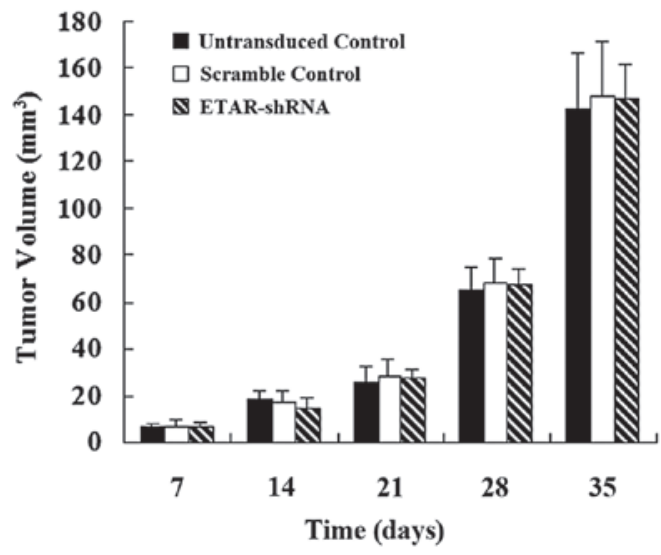

Figure 2. Tumor volume change over time in the orthotopic xenograft osteosarcoma mouse model. Untransduced MG-63 cells, or cells stably transduced with scramble control shRNA or ETAR-shRNA were injected into the left tibia of individual mice. The primary tumor volume was monitored. Results are expressed as the mean \pm SD. Thirty-five days after MG-63 inoculation, all animals were sacrificed, as the primary tumors had grown to a disabling size.

pairwise comparisons using the least significant difference method. The significance level of this study was set at twosided $\alpha=0.05$.

\section{Results}

MG-63 cells, an OS cell line constitutively expressing ET-1 and ETAR, were stably transduced with ETAR shRNA or scramble control shRNA. Western blot analysis showed that ETAR shRNA knocked down more than $75 \%$ of endogenous ETAR expression in MG-63 cells. In contrast, scramble control shRNA showed no significant effect on ETAR (Fig. 1A). In vitro cell proliferation and viability assay showed no significant difference in cell proliferation/viability among the untransduced control MG-63 cells and those stably transduced with scramble control shRNA or ETAR-shRNA (Fig. 1B). In vitro cell migration/invasion assays showed that cells stably transduced with ETAR-shRNA had a significantly lower number of migrated cells than the untransduced control cells or cells expressing scramble control shRNA (Fig. 1C).

To assess the role of ETAR in OS proliferation and pulmonary metastasis in vivo, we established an orthotopic xenograft OS mouse model. Untransduced MG-63 cells, or cells stably transduced with ETAR-shRNA or scramble control shRNA, were injected into the left tibia of individual mice. As shown in Fig. 2, no significant difference in the tumor volume was observed over time among the the ETAR-shRNA group and the control groups. In line with the in vitro data (Fig. 1B), the results suggest that the knockdown of ETAR had no significant effect on OS cell proliferation and viability in vivo.

Twenty-eight days after inoculation, $90 \%$ (9/10) of the mice in the untransduced control group, $80 \%(8 / 10)$ in the scramble control group, and $0 \%(0 / 10)$ in the ETAR-shRNA group exhibited obvious dyspnea and distress. The relevant mice in the untransduced control $(n=9)$ and the scramble control $(n=8)$ groups were sacrificed on Day 28. Eight randomly selected mice in the ETAR-shRNA group were sacrificed at the same time for group comparison of pulmonary metastasis. Compared with the ETAR-shRNA group, both the 
A

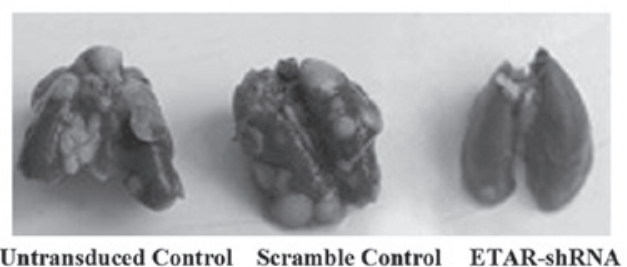

B

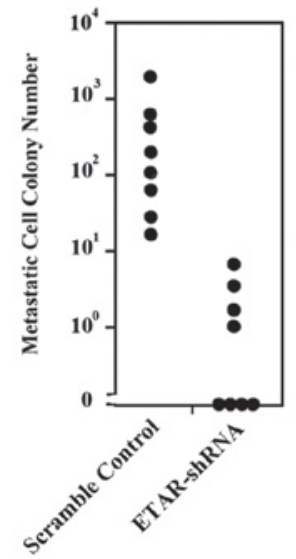

Figure 3. Pulmonary metastasis in the orthotopic xenograft osteosarcoma mouse model. (A) Typical lung images of the untransduced control, the scramble control and the ETAR-shRNA groups after fixation in Bouin solution for $24 \mathrm{~h}$. (B) Clonogenic lung metastasis assays were performed in the scramble control and the ETAR-shRNA groups 28 days after MG-63 inoculation. Each symbol represents a single animal ( $\mathrm{n}=8$ each group).

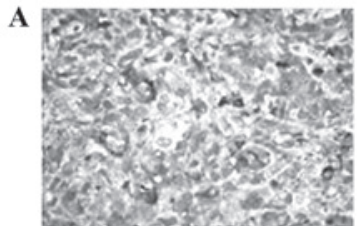

Normal Control

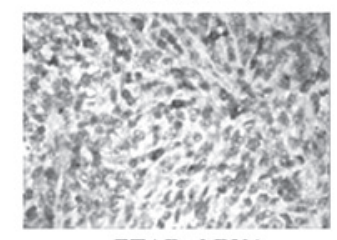

ETAR-shRNA

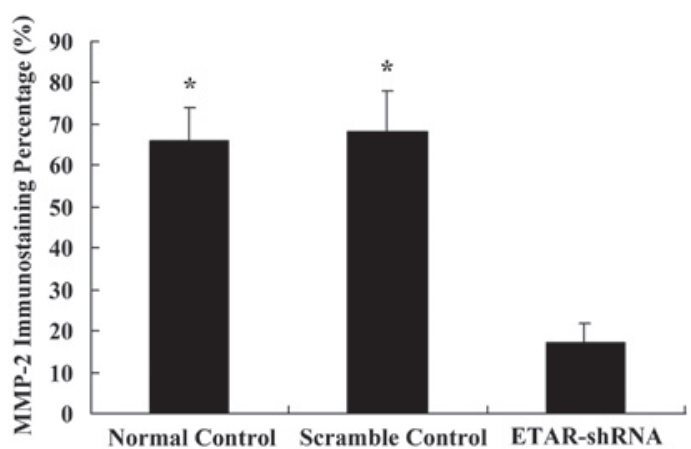

Figure 4. Immunohistochemical detection of matrix metalloproteinase-2 (MMP-2) expression in the primary tumor. (A) Immunohistochemical analyses were performed to determine MMP-2 expression in the primary tumors in the mouse model. Positive staining for MMP-2 was observed as a brownish color. Magnification, x200. (B) MMP-2-positive cells were counted and a percentage was calculated as follows: MMP-2-positive cells/total tumor cells x $100 \%$. The MMP-2 immunostaining percentage in the tumor was compared among the untransduced control, the scramble control and the ETAR-shRNA groups. ${ }^{*} \mathrm{P}<0.05$ compared with the ETAR-shRNA group
A

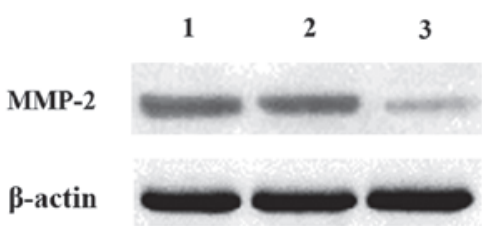

B

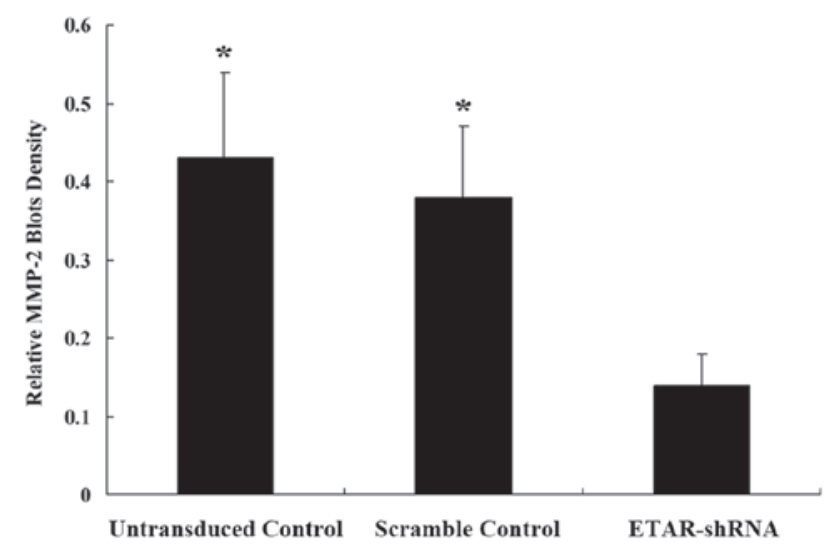

Figure 5. Western blot analysis of matrix metalloproteinase-2 (MMP-2) expression in the primary tumors. (A) Twenty-eight days after MG-63 inoculation, primary tumor tissue lysates from eight mice in the untransduced control (lane 1), the scramble control (lane 2) and the ETAR-shRNA (lane 3) groups were subjected to western blot analysis for MMP- 2 expression. $\beta$-actin blotting was used as a loading control. Representative results are shown in this figure. (B) MMP-2 and $\beta$-actin blots were measured by densitometry. Density of the MMP-2 blot was normalized against that of $\beta$-actin to obtain a relative MMP-2 blot density. * $\mathrm{P}<0.05$ compared with the ETAR-shRNA group.

A

MMP-2

$\beta$-actin

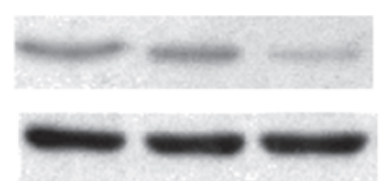

B

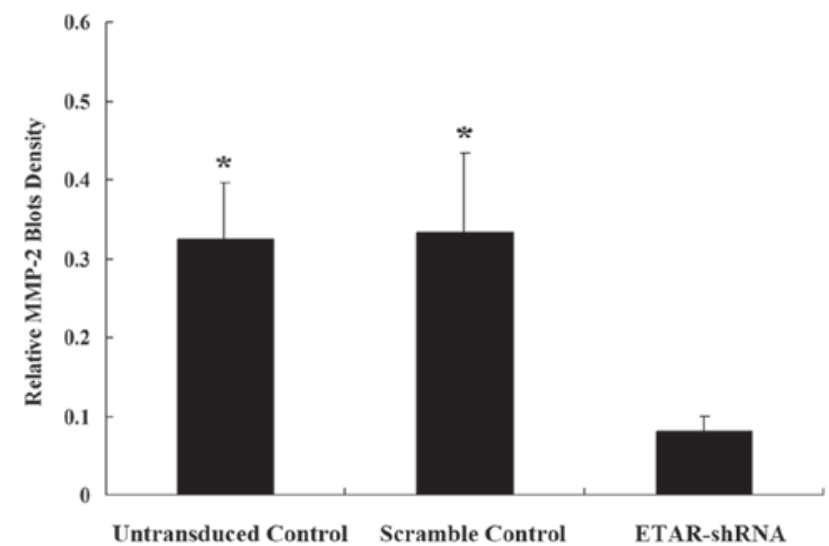

Figure 6. Western blot analysis of matrix metalloproteinase-2 (MMP-2) expression in stably transduced MG-63 cells. (A) Expression of MMP-2 in untransduced MG-63 cells (lane 1) and cells stably transduced with scramble control shRNA (lane 2) or ETAR-shRNA (lane 3) was analyzed with western blot analysis. $\beta$-actin blotting was used as a loading control. Representative results are shown in this figure. (B) MMP- 2 and $\beta$-actin blots were measured by densitometry. Density of the MMP-2 blot was normalized against that of $\beta$-actin to obtain a relative MMP-2 blot density. ${ }^{*} \mathrm{P}<0.05$ compared with the ETAR-shRNA cells. 
untransduced control and the scramble control groups showed markedly more metastatic nodules on the lung surface as well as more swollen and congested lungs (Fig. 3A). To quantitate the pulmonary metastasis, clonogenic lung metastasis assays were performed in the scramble control and the ETAR-shRNA groups based on puromycin selection. As shown in Fig. 3B, lung metastasis in the ETAR-shRNA group was significantly lower than that in the scramble control group, in line with the in vitro data (Fig. 1C).

ET-1 reportedly can induce the expression of MMP-2 and MMP-9 in OS cells, which are important promoters of tumor metastasis (15). To explore the mechanisms underlying the markedly reduced pulmonary metastasis in the ETAR-shRNA group, we examined the expression of different MMPs in the primary tumor on Day 28. Immunohistochemical analyses revealed that MMP-2 expression was significantly reduced in the ETAR-shRNA group compared with the control groups (Fig. 4). The results were confirmed with western blot analyses using primary tumor tissues (Fig. 5). Similar results were also observed in the stably transduced MG-63 cells (Fig. 6).

\section{Discussion}

In vitro studies suggest that ETAR is a potential therapeutic target for OS metastasis $(8,9)$. In the present study, we showed in an orthotopic xenograft OS mouse model that ETAR is critical for OS pulmonary metastasis, but not for tumor growth. This study provides the first in vivo evidence suggesting an important role for ETAR in OS pulmonary metastasis.

Our previous study showed that ETAR blockade inhibits OS cell invasion in vitro (9). In the present study, instead of blocking ETAR with an antagonist, we knocked down endogenous ETAR expression in xenograft OS cells to provide more direct evidence for the in vivo role of ETAR in OS proliferation and pulmonary metastasis. We used the MG-63 human osteosarcoma cell line in this study, since it constitutively expresses ET-1 and ETAR and has been used in previous in vitro studies $(8,9)$ on OS cell invasion.

The ET-1/ETAR pathway has been reported to promote tumor cell proliferation and viability in a variety of cancers, including ovarian cancer, hepatocellular cancer and prostate cancer (16-18). However, knockdown of ETAR expression failed to result in significant changes in OS growth both in vitro (Fig. 1B) and in vivo (Fig. 2) in the present study, suggesting that ETAR does not play an important role in OS proliferation/viability. This is in agreement with our previous results that treatment with an ETAR antagonist alone would not significantly affect the viability of OS cells in vitro (9). The underlying mechanism is still unclear.

In the present study, a combination of clinical signs, organ examinations and quantitative lung metastasis assays (Fig. 3) demonstrated that knockdown of ETAR expression in xenograft OS cells effectively inhibited OS pulmonary metastasis in the orthotopic xenograft OS mouse model. Our data showed that the knockdown of ETAR expression resulted in significant in vivo (Figs. 4 and 5) and in vitro (Fig. 6) downregulation of MMP-2, an important promoter of tumor metastasis by breaking down the extracellular matrix (15). This is in line with the findings of a previous in vitro study that ET-1 promotes MMP-2 induction in human OS cells (8). Thus, we propose that the important role of ETAR in OS pulmonary metastasis is at least partially mediated through its regulatory role in MMP-2 expression.

It is noteworthy that not all OS samples express ETAR (8). Thus, our findings are limited to those OS cells expressing ETAR. Nevertheless, our study for the first time demonstrates that ETAR is critical for OS pulmonary metastasis in vivo. This suggests that abrogating the function of ETAR either by selective antagonists or by gene therapy could effectively inhibit the pulmonary metastasis of OS cells that express ETAR.

\section{References}

1. Ottaviani G and Jaffe N: The epidemiology of osteosarcoma. Cancer Treat Res 152: 3-13, 2010.

2. Bacci G, Briccoli A, Rocca M, et al: Neoadjuvant chemotherapy for OS of the extremities with metastases at presentation: recent experience at the Rizzoli Institute in 57 patients treated with cisplatin, doxorubicin, and a high dose of methotrexate and ifosfamide. Ann Oncol 14: 1126-1134, 2003.

3. Kager L, Zoubek A, Potschger U, et al: Primary metastatic OS: presentation and outcome of patients treated on neoadjuvant Cooperative OS Study Group protocols. J Clin Oncol 21: 2011-2018, 2003.

4. Ta HT, Dass CR, Choong PF and Dunstan DE: Osteosarcoma treatment: state of the art. Cancer Metastasis Rev 28: 247-263, 2009.

5. Gorlick R, Anderson P, Andrulis I, et al: Biology of childhood osteogenic sarcoma and potential targets for therapeutic development: meeting summary. Clin Cancer Res 9: 5442-5453, 2003.

6. Wittig JC, Bickels J, Priebat D, et al: Osteosarcoma: a multidisciplinary approach to diagnosis and treatment. Am Fam Physician 65: 1123-1132, 2002.

7. Nelson J, Bagnato A, Battistini B and Nisen P: The endothelin axis: emerging role in cancer. Nat Rev Cancer 3: 110-116, 2003.

8. Felx M, Guyot MC, Isler M, Turcotte RE, Doyon J, Khatib AM, Leclerc S, Moreau A and Moldovan F: Endothelin-1 (ET-1) promotes MMP-2 and MMP-9 induction involving the transcription factor NF-kappaB in human osteosarcoma. Clin Sci (Lond) 110: 645-654, 2006

9. Zhao Y, Liao Q, Zhu Y and Long H: Endothelin-1 promotes osteosarcoma cell invasion and survival against cisplatin-induced apoptosis. Clin Orthop Relat Res 469: 3190-3199, 2011.

10. Yu L, Wang CY, Miao L, Du X, Mayer D and Zhang J: Estrogens promote invasion of prostate cancer cells in a paracrine manner through up-regulation of matrix metalloproteinase 2 in prostatic stromal cells. Endocrinol 152: 773-781, 2011.

11. Dass CR, Ek ET, Contreras KG and Choong PF: A novel orthotopic murine model provides insights into cellular and molecular characteristics contributing to human osteosarcoma. Clin Exp Metastasis 23: 367-380, 2006.

12. Ek ETH, Dass CR, Contreras KG and Choong PFM: Inhibition of orthotopic osteosarcoma growth and metastasis by multitargeted antitumour activities of pigment epithelium-derived factor. Clin Exp Metastasis 24: 93-106, 2007.

13. Pilones KA, Kawashima N, Yang AM, Babb JS, Formenti SC and Demaria S: Invariant natural killer T cells regulate breast cancer response to radiation and CTLA-4 blockade. Clin Cancer Res 15: 597-606, 2009

14. Sun P, Xiong H, Kim TH, Ren B and Zhang Z: Positive interregulation between beta-catenin/T cell factor- 4 signaling and endothelin-1 signaling potentiates proliferation and survival of prostate cancer cells. Mol Pharmacol 69: 520-531, 2006.

15. Sato $\mathrm{H}$ and Takino T: Coordinate action of membrane-type matrix metalloproteinase-1 (MT1-MMP) and MMP-2 enhances pericellular proteolysis and invasion. Cancer Sci 101: 843-847, 2010.

16. Bagnato A, Salani D, Di Castro V, et al: Expression of endothelin 1 and endothelin A receptor in ovarian carcinoma: evidence for an autocrine role in tumor growth. Cancer Res 59: 720-727, 1999.

17. Nakamuta M, Ohashi M, Tabata S, et al: High plasma concentrations of endothelin-like immunoreactivities in patients with hepatocellular carcinoma. Am J Gastroenterol 88: 248-252, 1993.

18. Nelson JB, Hedican SP, George DJ, et al: Identification of endothelin-1 in the pathophysiology of metastatic adenocarcinoma of the prostate. Nat Med 1: 944-949, 1995. 